\title{
Animal Classification using Deep Learning Inception-v3 Model on Tensorflow
}

\author{
Chetana Khandale ${ }^{1}$, Mayank Ramdev ${ }^{2}$ \\ Department of Computer Science and Engineering, Sagar Institute of Research and Technology, Bhopal, India ${ }^{1,2}$
}

\begin{abstract}
Identification of similar types of objects in image processing now become regular task, but when dissimilar objects come into picture it becomes quite complex. Even it become more complicated when not just we need to identify objects but also categorizes them into their specific classes. We are using machine learning technique to classify the animal and put them into specified classes. Inception-v3 is the open source algorithm made by Google and used for objects classification. In this paper, based on Inception-v3 model in TensorFlow platform, we use the transfer learning technology to retrain the animal category datasets, which can greatly improve the accuracy of animal classification.
\end{abstract}

Keywords: Classification, Inception-v3, image processing, TensorFlow

\section{INTRODUCTION}

Zoology is the study of animals. Currently there are over 66 thousand (less than 5\% of all animals) vertebrate species, and over 1.3 million (over $95 \%$ of all animals) invertebrate species in existence. Species identification and its classification is a fundamental research work in the zoology [18]. When human eyes see the mammals it can easily identify what exactly it is but when it comes to embedded vision devices it become complex task. Therefore, having a fast and accurate animal classifier will bring a lot of eagerness in peoples' lives. There are some challenges in mammal classification like the similarity between the different species of animals, the complex background of animal image. We cannot just rely on a single feature, such as colour, texture or shape to distinguish them. The same animal will be different because of the shades of colours, shape, scale, view point etc.

In 2012 ImageNet Large Scale Visual Recognition Competition (ILSVRC) [11] Convolutional Neural Networks (CNN) have become more popular than AlexNet in computer vision after winning the competition [12] [10]. To achieve higher accuracy in image classification development and usage of deeper and complex CNN become trend in research [13] [16] [14] [8]. However, the complex structure of models does not to not necessarily making networks more efficient with respect to speed and size to increase the accuracy. On computationally limited platform object detection and recognition has to be done with time critical manner, for example online games, self-driving car, robotics and automation, augmented reality [7].

Convolutional neural network is an efficient recognition method which has been developed in recent years. This network avoids the complex pre-processing of the image, and people can input the original image directly. It uses local receptive field, weights sharing and pooling technology and makes the training parameters greatly reduced compared to the neural network [2] [6]. It also has a certain degree of translation, rotation and distortion invariance of image. It has made great progress in the field of image classification. TensorFlow [1] is the second generation of artificial intelligence learning system developed by Google, which supports the convolutional neural network (CNN), recurrent neural network (RNN) and other depth of the neural network model, which can be used in speech recognition, image recognition and so on many machines deep learning field.

In this paper we have shown a mammals' classification using machine learning pertained model Inception v-3. In mammals we are using classes as Lion, Tiger, Elephant, Giraffe, and Monkey. Section II describes existing work with TensorFlow in constructing small models. Section III describes main theme, the Inception-v3 architecture. Section IV gives building of classification model; Section $\mathrm{V}$ refer to experimentation on five different mammals' datasets. Section VI concludes with summary.

\section{EXISTING WORK}

Compared with the traditional image classification methods, convolutional neural network use multilayer convolution to extract features and combine the features automatically. It has a higher recognition rate and a wider range of applications. 


\title{
International Journal of Advanced Research in Computer and Communication Engineering
}

\author{
Vol. 8, Issue 2, February 2019
}

TensorFlow [1] as the second generation of Google artificial intelligence learning system has got much attention and affirmation in the field of the machine learning in all over the world. TensorFlow has ranked first in all machine learning and deep learning programs so far. It has the advantages of high availability and high flexibility, and with the support of TensorFlow researchers, the efficiency of TensorFlow is improved. Today, Google has released a number of pretrained models on the TensorFlow's official website, to facilitate the use of researchers in different fields.

Inception-v3 [3] is one of the pretrained models on the TensorFlow. It is a rethinking for the initial structure of computer vision after Inception-v1 [5], Inception-v2 [4] in 2015. The Inception-v3 [3] model is trained on the ImageNet datasets, containing the information that can identify 1000 classes [11] [13]. In ImageNet, the error rate of top-5 is $3.5 \%$, the error rate of top-1 dropped to $17.3 \%$. Tensorflow [1] also provides detailed tutorials for us to retrain Inception's final Layer for new categories using transfer learning.

Transfer learning is a new machine learning method which can use the existing knowledge learned from one environment and solve the other new problem which is different but has some relation with the old problem. For example, we can apply the knowledge learned from the motorcycle problem to the study of bike problem. Compared with the traditional neural network, it only needs to use a small amount of data to train the model, and achieve high accuracy with a short training time.

\section{CONSTRUCTION OF ANIMAL CLASSIFICATION MODEL}

This section focuses on experimental setup for mammals' classification model using Inception-v3 on Tensor Flow framework. Here classification model is separated into following four stages: image preprocessing, training, verification followed by testing.

\section{A. Image Preprocessing}

Image pre-processing is that the name for operations on pictures at all-time low level of abstraction whose aim is associate degree improvement of the image information that suppress unwanted distortions or enhances some image features important for further processing. It does not increase image information content. Its methods use the considerable redundancy in images. Neighbouring pixels corresponding to one object in real images have the same or similar brightness value and if a distorted pixel can be picked out from the image, it can be restored as an average value of neighbouring pixels.

\section{B. Training Process}

While training the model we use approximately 2000 image dataset, around 400 images per mammal, every image is used multiple times through training process. Computing the layers behind the layer just before the final output layer which performs the grouping for each image takes a substantial time. As the lower layers of the network are not being changed their outputs can be stored and used again.

\section{Verification and testing process}

By testing, we mean evaluating the system in several conditions and observing its behavior, as stated above we are not just providing single image as input to the inception model instead multiple images multiple times watching for defects. By verification, we mean producing a compelling argument that the system will not misbehave under a very broad range of circumstances so the accuracy of model will not be varied.

\section{EXPERIMENT}

To complete this work, we used python 3.5, Tensorflow 1.2 and the hardware platform is Lenovo G50 laptop: processor $1.90 \mathrm{GHz}$ Intel i3, memory 4GB.

In the proposed system, the mammals' image dataset is collected from google and class wise batches are created. This dataset is used as input to the machine learning model in which retraining will be provided based upon pretrained inception v3 algorithm [16]. TensorBoard is a monitoring and inspection tool included with tensorflow. We use it to monitor the training progress. Once the training is done sample input image is used for testing and validation on which the accurate recognition will be done [17]. 
Vol. 8, Issue 2, February 2019

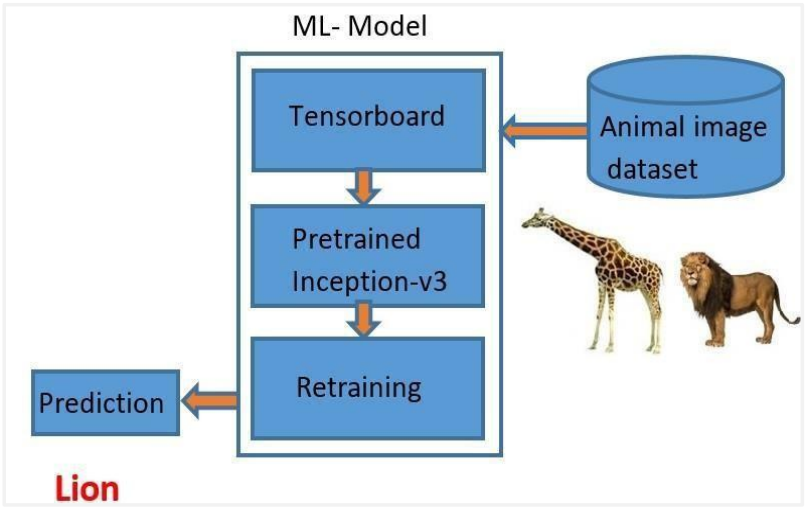

Figure: 1. System Architecture

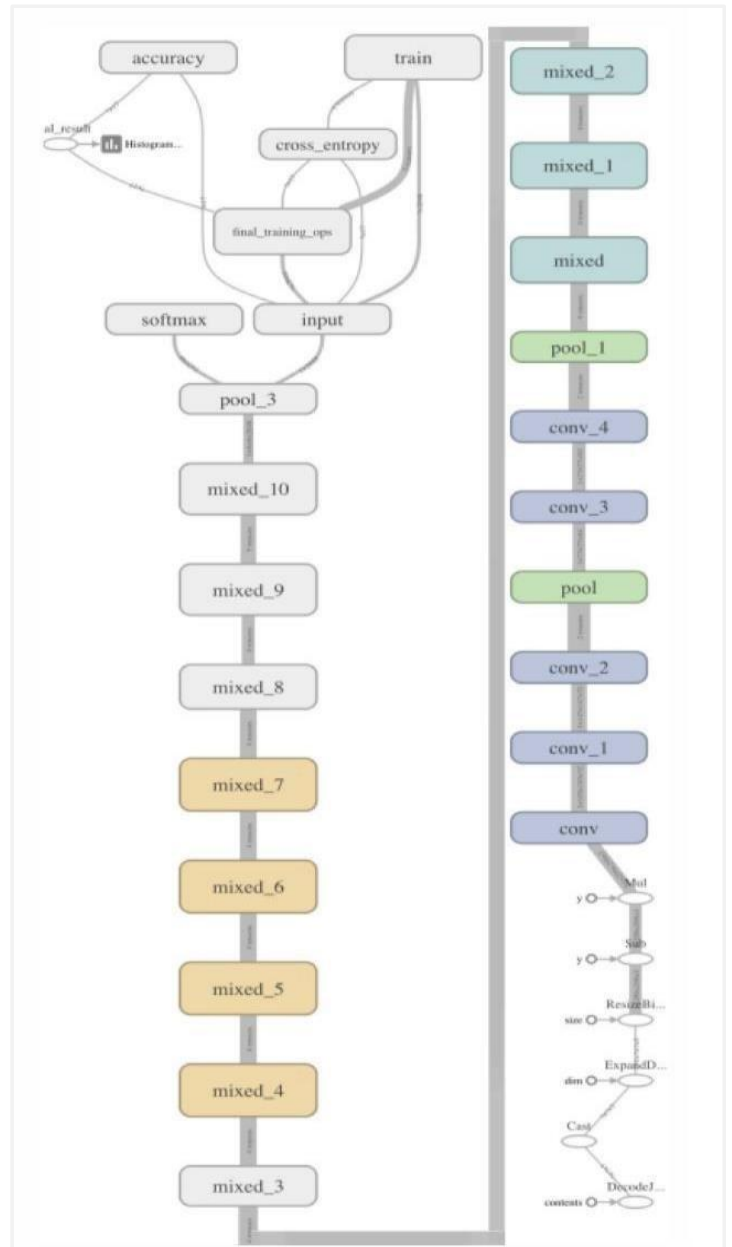

Figure: 2. Main graph of Inception-v3 model

A. Procedure of Inception-v3

In Inception-v3, transfer learning method is used. We should keep the parameters of the previous layer, then remove the last layer and input the mammal dataset to retrain the new last layer, the number of output nodes will be changed to 5 because we used only 5 different mammals. The last layer of the model is trained by back propagation algorithm, and the cross entropy cost function is used to adjust the weight parameter by calculating the error between the output of the softmax layer and the label vector of the given sample category [3] [15]. 


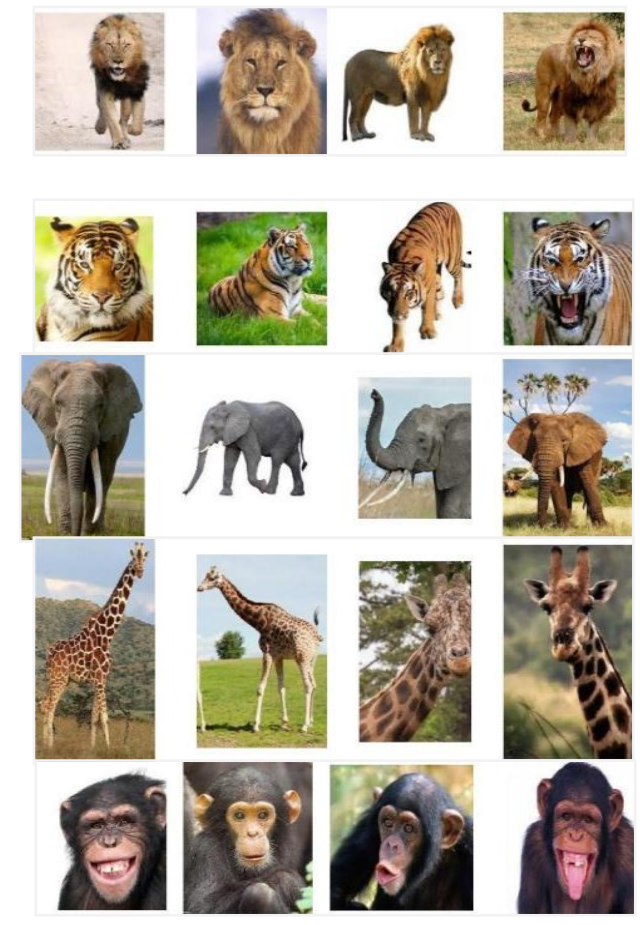

Figure: 3. Sample image dataset

\section{RESULTS}

Figure 4 and figure 5 show the variation of accuracy and cross entropy based on mammals' dataset. The orange line represents the training set, and the green line represents the validation set.

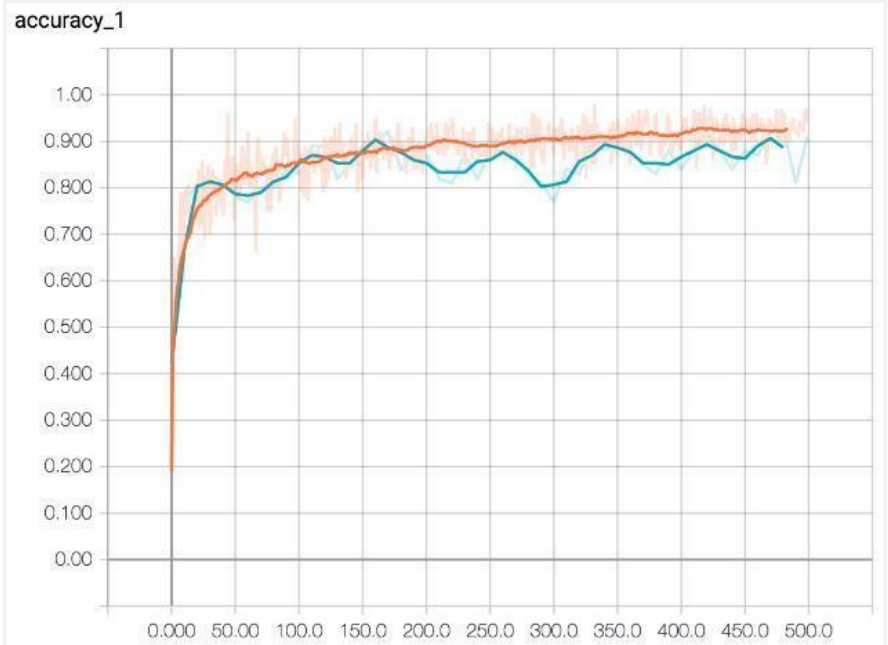

Figure: 4. the variation of accuracy on mammals' dataset

The training accuracy shows the share of the photographs utilized in this training batch that were tagged with the proper class label. Validation accuracy: The validation accuracy is that the exactness (percentage of correctly-labelled pictures) on a randomly-selected cluster of images from a unique set. 


\section{International Journal of Advanced Research in Computer and Communication Engineering}

Vol. 8, Issue 2, February 2019

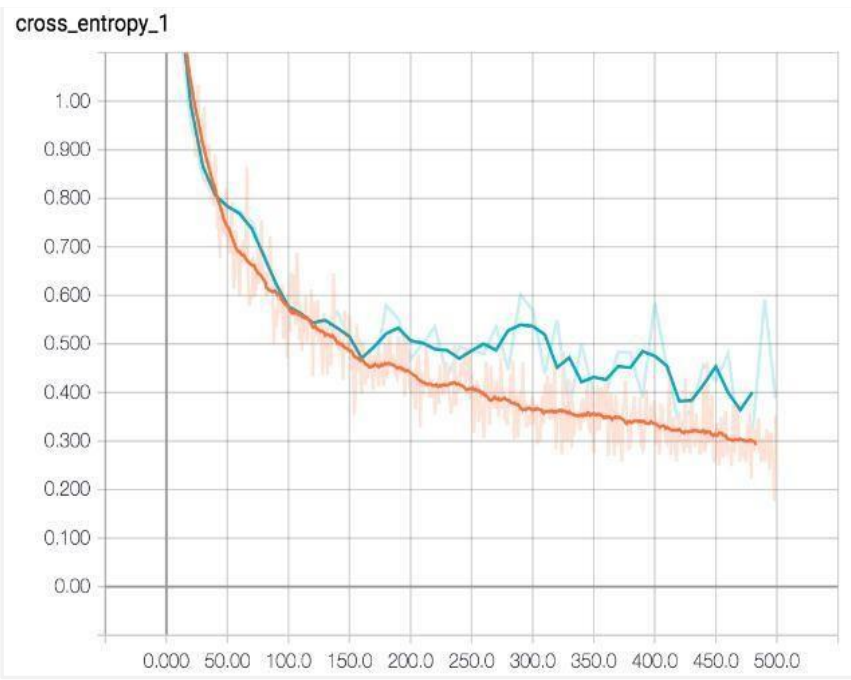

Figure 5. The variation of cross entropy on mammals' dataset

Cross entropy is a loss function that gives a glimpse into how well the learning process is progressing (lower numbers are better here)

Table 1: Description of the four figures

\begin{tabular}{|l|l|}
\hline Index & Accuracy \\
\hline accuracy of training set & $99 \%-100 \%$ \\
\hline accuracy of validation set & $95 \%$ \\
\hline cross entropy of training set & 0.10 \\
\hline cross entropy of validation set & 0.24 \\
\hline
\end{tabular}

\section{CONCLUSION}

We proposed the system based on the Inception-v3 model of TensorFlow platform, in which we use the transfer learning technology to train an animal classification model on mammals' dataset. The classification accuracy of the model is approximately $95 \%$ on given dataset, which is higher than other method available for classification. The future work is to study and develop a more effective and accurate model for image classification.

\section{REFERENCES}

[1]. Peter Goldsborough Fakultät für Informatik, "A Tour of TensorFlowarXiv:1610.01178v1 [cs.LG] 1 Oct 2016.

[2]. Abhineet Saxena, "Convolutional neural networks: an illustration in TensorFlow". ACM Crossroads 22(4): 56-58, 20 16.

[3]. Nitin R. Gavai; Yashashree A. Jakhade; Seema A. Tribhuvan; Rashmi Bhattad "MobileNets for Flower Classification using TensorFlow" International Conference on Big Data, IoT and Data Science(BID) 10.1109/BID.2017.8336590, pg154 - 158, IEEE, 2017.

[4]. Xiaoling Xia, Cui Xu, Bing Nan, "Inception-v3 for Flower Classification," 2nd International Conference on Image, Vision and Computing, 2017.

[5]. J. Krause, B. Sapp, A. Howard, H. Zhou, A. Toshev, T. Duerig, J. Philbin, and L. Fei-Fei."The unreasonable effectiveness of noisy data for fine-grained recognition". arXiv preprint arXiv:1511.06789, 2015.

[6]. Sergey loffe, Christian Szegedy, et al. "Batch Normalization: Accelerating Deep Network Training by Reducing Internal CovarIate Shift". ICML20 15: 448-456, 2015.

[7]. J. Christian Szegedy, Wei Liu, et aI, “Going Deeper with Convolutions”. arXiv:1409.4842,2014.

[8]. Angelova A, Zhu S, Efficient Object Detection and Segmentation for Fine-Grained Recognition[C]. Computer Vision and Pattern Recognition: 81 1-8 18, IEEE, 2013.

[9]. K. He, X. Zhang, S. Ren, and J. Sun. Deep residual learning for image recognition. arXiv preprint arXiv:1512.03385, 2015.

[10]. Maria-Elena Nilsback, Andrew Zisserman: Automated Flower Classification over a Large Number of Classes. ICVGlP 2008: 722 - 729, 2008.

[11]. F. N. Iandola, M. W. Moskewicz, K. Ashraf, S. Han, W. J. Dally, and K. Keutzer. Squeezenet: Alexnetlevel accuracy with 50x fewer parameters and 1 mb model size. arXiv preprint arXiv:1602.07360, 2016.

[12]. Krizhevsky, I. Sutskever, and G. E. Hinton. Imagenet classification with deep convolutional neural networks. In Advances in neural information processing systems, pages 1097-1105, 2012

[13]. O. Russakovsky, J. Deng, H. Su, J. Krause, S. Satheesh, S. Ma, Z. Huang, A. Karpathy, A. Khosla, M. Bernstein, et al. Imagenet large scale visual recognition challenge. International Journal of Computer Vision, 115(3):211-252, 2015 


\title{
International Journal of Advanced Research in Computer and Communication Engineering
}

\author{
Vol. 8, Issue 2, February 2019
}

[14]. K. Simonyan and A. Zisserman. Very deep convolutional networks for large-scale image recognition. arXiv preprint arXiv:1409.1556, 2014.

[15]. C. Szegedy, S. Ioffe, and V. Vanhoucke. Inception-v4, inception-resnet and the impact of residual connections on learning. arXiv preprint arXiv: $1602.07261,2016$

[16]. C. Szegedy, W. Liu, Y. Jia, P. Sermanet, S. Reed, D. Anguelov, D. Erhan, V. Vanhoucke, and A. Rabinovich. Going deeper with convolutions. In Proceedings of the IEEE Conference on Computer Vision and Pattern Recognition, pages 1-9, 2015.

[17]. C. Szegedy, V. Vanhoucke, S. Ioffe, J. Shlens, and Z. Wojna. "Rethinking the inception architecture for computer vision". arXiv preprint arXiv:1512.00567, 2015.

[18]. K. He, X. Zhang, S. Ren, and J. Sun. "Deep residual learning for image recognition". arXiv preprint arXiv:1512.03385, 2015.

[19]. Merriam-Webster's, "Animals". Retrieved 16 May 2010. 2 a: one of the lower animals as distinguished from human beings b: mammal; broadly: vertebrate" 